\title{
Gastrointestinal stromal tumor with nephrotic syndrome as a paraneoplastic syndrome: a case report
}

\author{
Kiyoko Takane ${ }^{1}$, Yutaka Midorikawa ${ }^{1 *}$, Shintaro Yamazaki ${ }^{1}$, Takahiro Kajiwara ${ }^{1}$, Naoki Yoshida ${ }^{1}$, Yoshiaki Kusumi ${ }^{2}$ \\ and Tadatoshi Takayama'
}

\begin{abstract}
Introduction: Paraneoplastic syndromes are disorders associated with clinical signs and symptoms caused by substances produced by malignant disease and are not directly related to the physical effects of a primary or metastatic tumor. We describe a patient with gastrointestinal stromal tumor of the stomach accompanied by nephrotic syndrome as paraneoplastic syndrome in whom symptomatic treatment was ineffective. Nephrotic syndrome caused by gastrointestinal stromal tumors is quite rare, and to the best of our knowledge this is the first time that such a case has been documented.

Case presentation: We describe a 69-year-old Asian woman with a gastrointestinal stromal tumor of the stomach accompanied by paraneoplastic syndrome. The patient had severe hypoalbuminemia and proteinuria, which were apparently attributed to a gastrointestinal stromal tumor. After preoperative treatment for hypoalbuminemia, the tumor was resected and nephrotic syndrome improved. Two years after her operation, she is still alive with neither tumor recurrence nor nephrotic syndrome.

Conclusion: Patients with refractory nephrotic syndrome caused by a malignant tumor should be treated aggressively, even if they are in poor general condition. Otherwise, the opportunity for potentially curative surgery may be missed.
\end{abstract}

Keywords: Gastrointestinal stromal tumor, Nephrotic syndrome, Paraneoplastic syndrome

\section{Introduction}

Paraneoplastic syndrome (PNS) is a disorder or symptom caused by cancer or a reaction to a tumor, but is not due to the local presence of cancer cells [1,2]. PNS can resolve after complete tumor resection or a response to chemotherapy and recur in association with tumor recurrence. Clinical manifestations are mediated by hormones or cytokines excreted by tumor cells or by an immune response against the tumor [3].

The signs and symptoms of PNS are diverse, but common features include neuropathy, skin disease, and nephrotic syndrome. Peripheral neuropathy is one of the most frequent types of PNS of the nervous system and is

\footnotetext{
* Correspondence: mido-tky@umin.ac.jp

${ }^{1}$ Department of Digestive Surgery, Nihon University School of Medicine, 30-1

Oyaguchi Kami-machi, Itabashi-ku, Tokyo 173-8610, Japan

Full list of author information is available at the end of the article
}

usually associated with slight loss of muscle strength, hypoesthesia, and areflexia [4]. Itching paresthesia is the most common cutaneous symptom, and such symptoms may improve after treatment of the primary disease [1]. PNS is common among middle-aged and elderly patients who harbor cancers of the lung, breast, or ovaries, or lymphoma as the primary disease [5].

We describe a patient with gastrointestinal stromal tumor (GIST) of the stomach accompanied by nephrotic syndrome as PNS in whom symptomatic treatment was ineffective, but clinical remission was obtained after an operation. To the best of our knowledge, this is the first time such a case has been documented. We also discuss the incidence, diagnosis, and treatment of GIST accompanied by PNS. 


\section{Case presentation}

A 69-year-old Asian woman was admitted to our hospital because of diminished appetite. She had no previous medical history and was not complaining of fever and, therefore, she was not taking oral non-steroid antiinflammatory drugs. Computed tomography revealed a gastric tumor measuring $56 \times 55 \mathrm{~mm}$, accompanied by calcification (Figure 1). Angiography via her left gastric artery showed staining of the tumor (Figure 2), and endoscopic ultrasonography demonstrated a heterogeneous lesion $60 \mathrm{~mm}$ in diameter, arising in the posterior wall of the stomach (Figure 3). Laboratory examinations revealed hypoproteinemia $(4.8 \mathrm{~g} / \mathrm{dL}$; normal range, 6.5 to $8.0)$, hypoalbuminemia $(2.2 \mathrm{~g} / \mathrm{dL}$; normal range, 3.8 to $5.3)$, and severe proteinuria $(8.47 \mathrm{~g} / \mathrm{dL}$; normal range, 0 to 0.15 ), and her protein creatinine ratio was $14.3 \mathrm{~g} / \mathrm{g}$ and amount of urine for 24 hours was $1200 \mathrm{~mL}$, clinically suggesting nephrotic syndrome. Therefore, to avoid an invasive inspection, a kidney biopsy was not carried out. There were no neurologic symptoms or skin disease.

Despite albumin administration, her hypoalbuminemia did not improve preoperatively. She was given a diagnosis of GIST of the stomach accompanied by nephrotic syndrome, and partial gastrectomy was performed. Macroscopically, a well-demarcated, hard, whitish submucosal tumor arose from the gastric wall (Figure 4a); histological and immunohistochemical examinations revealed a spindle-cell tumor positive for CD34 and ckit (Figure 4b, 4c), and a GIST was diagnosed.

Her postoperative course was uneventful. One week after her operation, her serum albumin $(3.3 \mathrm{~g} / \mathrm{dL})$ and urinary protein $(1.71 \mathrm{~g} / \mathrm{dL})$ levels remarkably improved after removal of the tumor. She remains well with no recurrence of the tumor or nephrotic syndrome (her recent urinary protein level: $1.19 \mathrm{mg} / \mathrm{mL}$ ) 2 years after surgery.

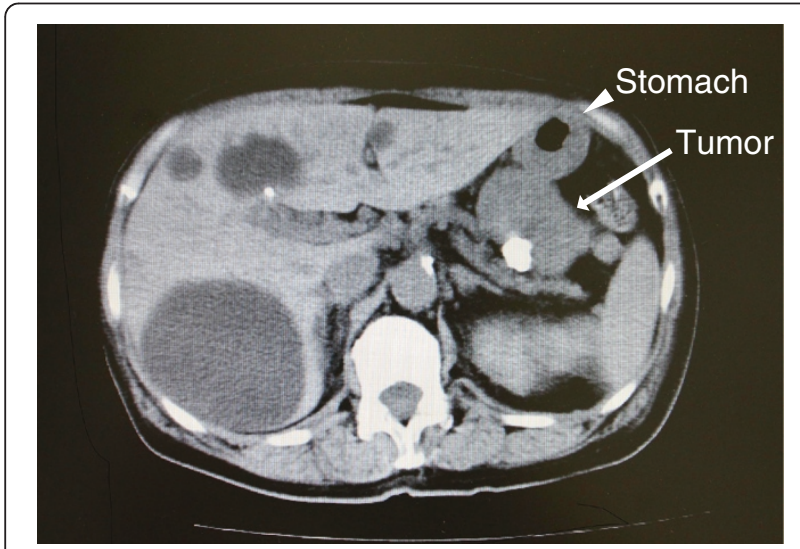

Figure 1 Computed tomographic findings of the tumor. A computed tomographic scan showing a large tumor measuring $56 \times 55 \mathrm{~mm}$ accompanied by calcification. An arrow indicates the tumor; the arrow head indicates the stomach.

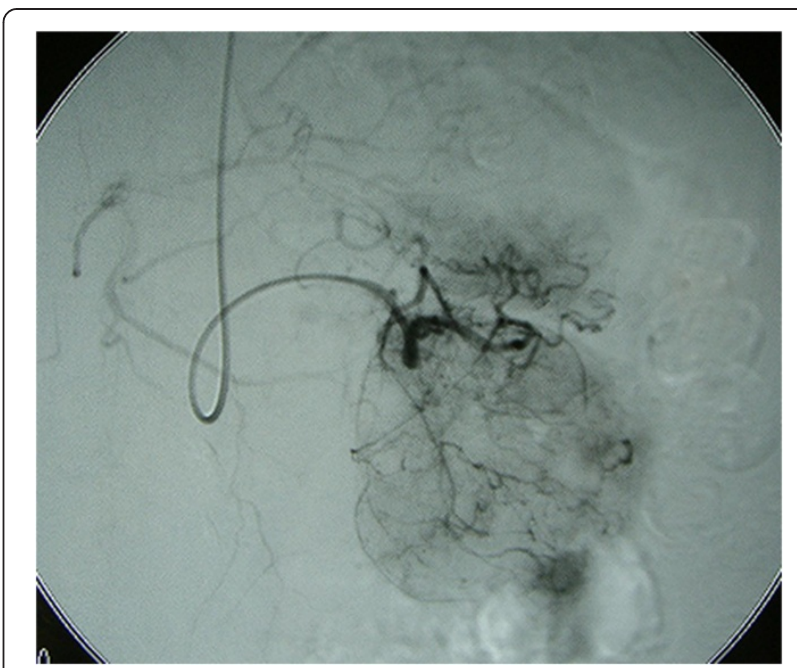

Figure 2 Angiographic findings of the tumor. Angiography via the left gastric artery showed staining of the tumor.

\section{Discussion}

Nephrotic syndrome can occur as malignancy-associated PNS, and previous studies have estimated that cancer occurs in $11 \%$ to $22 \%$ of patients with nephrotic syndrome $[2,6,7]$. Therefore, if an adult presents with nephrotic syndrome, physicians should bear in mind the risk of underlying malignant disease. Gastric cancer, lung cancer, and malignant lymphoma are frequently associated with nephrotic syndrome of which the rates are 25 , 15 and $10 \%$, respectively [8]. To the best of our knowledge, GIST has not been previously reported as an

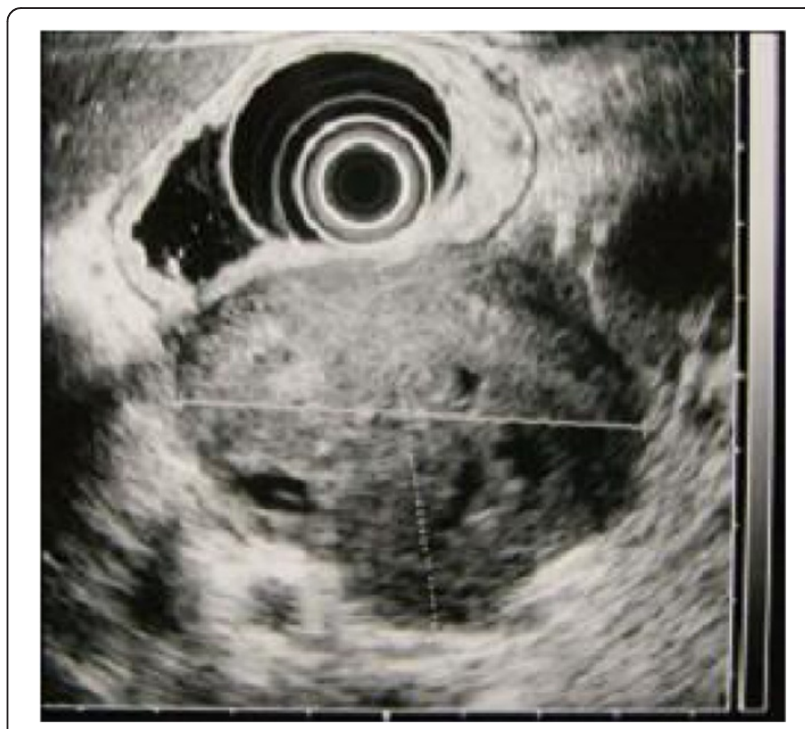

Figure 3 Endoscopic ultrasonographic findings of the tumor. Endoscopic ultrasonography demonstrated a heterogeneous lesion $60 \mathrm{~mm}$ in diameter, arising in the posterior wall of the stomach. 

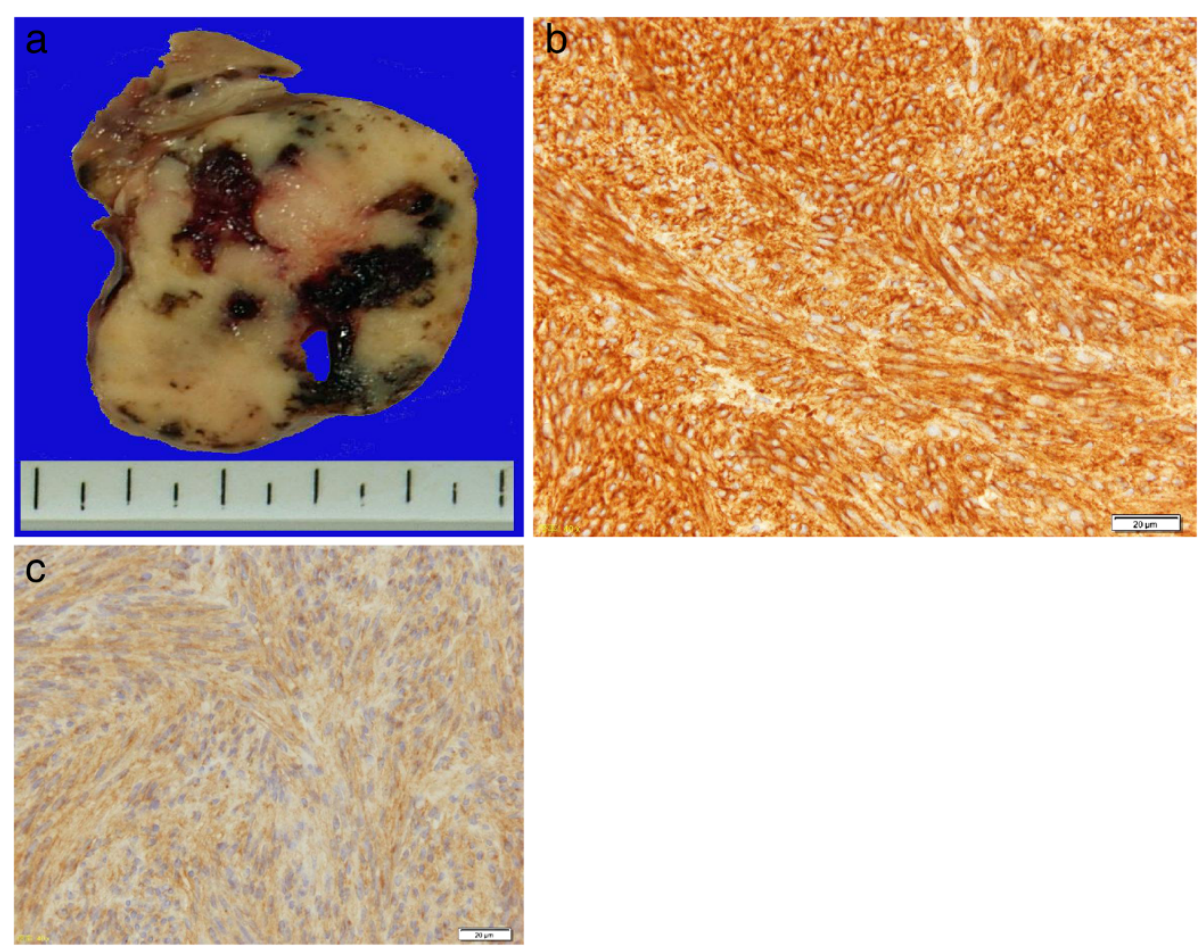

Figure 4 Pathological findings of the tumor. (a) Macroscopically, the tumor appeared to be gray-whitish and hard and arose from the gastric wall. (b,c) Both CD34 and c-kit were positive on immunohistochemical staining.

underlying disease of PNS, and this is the first such case to be documented.

In patients with PNS, surgical tumor removal can be followed by the regression of nephrotic syndrome. One study reported that nephrotic syndrome improved after operation in $78 \%$ of patients [4]. Therefore, surgical resection should be performed even in the presence of poor general condition due to PNS, because cancer is a potential cause of nephrotic syndrome [9]. The hypoalbuminemia and proteinuria in our patient did not preoperatively respond to symptomatic treatment. By contrast, clinical remission of nephrotic syndrome was obtained immediately after tumor removal. Considering that the patient did not take drugs perioperatively which cause nephrotic syndrome, such as non-steroidal anti-inflammatory drugs or tyrosine kinase inhibitors, the nephrotic syndrome was PNS associated with a GIST of the stomach.

Hypothetical explanations have been proposed to link minimal-change glomerulopathy to solid tumors. One potential mechanism involves the secretion of some tumoral factor that increases glomerular permeability without affecting glomerular capillary structure, resulting in massive proteinuria. Alternatively, tumor-specific antigens such as carcinoembryonic antigens may react with antibodies to form immune complexes, which accumulate as deposits in the glomerular basement membrane and cause renal dysfunction. The mechanism of this phenomenon can involve type III allergy [10].

In summary, we have documented a case of GIST of the stomach that was associated with nephrotic syndrome as PNS. Patients with refractory nephrotic syndrome caused by a malignant tumor should be treated aggressively to improve signs and symptoms associated with PNS. Otherwise, the opportunity for potentially curative surgery may be lost.

\section{Conclusions}

Nephrotic syndrome caused by GISTs is quite rare, and to the best of our knowledge this is the first time that such a case has been documented. Patients with refractory nephrotic syndrome caused by a malignant tumor should be treated aggressively, even if they are in poor general condition. Otherwise, the opportunity for potentially curative surgery may be missed.

\section{Consent}

Written informed consent was obtained from the patient for publication of this case report and any accompanying images. A copy of the written consent is available for review by the Editor-in-Chief of this journal. 


\section{Abbreviations}

GIST: Gastrointestinal stromal tumor; PNS: Paraneoplastic syndrome.

\section{Competing interests}

The authors declare that they have no competing interests.

\section{Authors' contributions}

YM was a major contributor in writing the manuscript. TK, NY, SY and TT performed the surgical procedure and obtained the patient data. YK performed the pathologic examination and was a contributor in writing the manuscript. All authors read and approved the final manuscript.

\section{Acknowledgements}

We acknowledge the support of all members of the surgical staff in Nihon University School of Medicine.

This work was mainly supported by a Grant-in-Aid for Scientific Research (A) 24249068 (T.T.) and (C) 23591972 (Y.M.) from the Ministry of Education, Culture, Sports, Science and Technology (MEXT), Japan.

\section{Author details}

'Department of Digestive Surgery, Nihon University School of Medicine, 30-1 Oyaguchi Kami-machi, Itabashi-ku, Tokyo 173-8610, Japan. ${ }^{2}$ Department of Pathology, Nihon University School of Medicine, 30-1 Oyaguchikami-machi, Itabashi-ku, Tokyo 173-8610, Japan.

Received: 19 October 2013 Accepted: 5 February 2014

Published: 27 March 2014

\section{References}

1. Yamauchi H, Linsey MS, Biava CG, Hopper I Jr: Cure of membranous nephropathy after resection of carcinoma. Arch Int Med 2061, 1985:145.

2. Lee JC, Yamauchi H, Hopper J Jr: The association of cancer and the nephrotic syndrome. Ann Intern Med 1966, 64:41-51.

3. Darnell RB, Posner JB: Paraneoplastic syndromes involving the nervous system. N Engl J Med 2003, 349:1543-1554.

4. Ikenaga S, Oishi S, Tsutsumi S, Norihito K, Makoto N, Hideki M, Takaaki Y, Hiroshi T: A case of nephrotic syndrome cured by removal of the advanced gastric cancer. in Japanese with English abstract) Nihon Shokakibyo Gakkai Zasshi (Jpn J Gastroenterol 2009, 42:489-494.

5. Kumar V, Abbas AK, Fausto N, Mitchell R: Robbins Basic Pathology. 8th edition. Philadelphia: Saunders; 2007.

6. Row PG, Cameron JS, Turner DR, Evans DJ, White RH, Ogg CS, Chantler C, Brown CB: Membranous nephropathy. Long-term follow-up and association with neoplasia. Q J Med 1975, 44:207-239.

7. Zech P, Colon S, Pointet P, Deteix P, Labeeuw M, Leitienne P: The nephrotic syndrome in adults aged over 60: etiology, evolution and treatment of 76 cases. Clin Nephrol 1982, 17:232-236.

8. Keur I, Krediet RT, Arisz L: Glomerulopathy as a paraneoplastic phenomenon. Neth J Med 1989, 34:270-284.

9. Meyrier A, Delahousse M, Callard P, Rainfray M: Minimal change nephrotic syndrome revealing solid tumors. Nephron 1992, 61:220-223.

10. Wakashin M, Wakashin Y, lesato K, Ueda S, Mori Y, Tsuchida H, Shigematsu $\mathrm{H}$, Okuda K: Association of gastric cancer and nephrotic syndrome. An immunologic study in three patients. Gastroenterology 1980, 78:749-756.

\section{doi:10.1186/1752-1947-8-108}

Cite this article as: Takane et al:: Gastrointestinal stromal tumor with nephrotic syndrome as a paraneoplastic syndrome: a case report. Journal of Medical Case Reports 2014 8:108.

\section{Submit your next manuscript to BioMed Central and take full advantage of:}

- Convenient online submission

- Thorough peer review

- No space constraints or color figure charges

- Immediate publication on acceptance

- Inclusion in PubMed, CAS, Scopus and Google Scholar

- Research which is freely available for redistribution

Submit your manuscript at www.biomedcentral.com/submit
C Biomed Central 